\title{
Removal of an Extensive Nasal Polyp in a Standing Horse, Using a Direct Approach to the Nasal Cavity Through a Bone Flap
}

\author{
Cristian Mihăiță CRECAN, Iancu MORAR, Mirela Alexandra RUS*, Marian TAULESCU, Liviu \\ OANA, Cosmin Petru PEȘTEAN ${ }^{*}$
}

Faculty of Veterinary Medicine, University of Agricultural Sciences and Veterinary Medicine, Cluj-Napoca, Romania
* Corresponding author: Cristian Mihăiță CRECAN e-mail: mirela.tripon@ usamvcluj.ro

\section{SHORT COMMUNICATION}

\begin{abstract}
Nasal polyps are not very common in horses, comprising of just about 2,5\% of equine sinonasal disease (Tremaine and Dixon, 2001). However, nasal polyps can develop into extensive masses, obstructing partially or totally air flow thus giving dramatic clinical presentations. The aim of this report was to present a surgical technique used to successfully remove a massive nasal polyp in a standing mare. A 9 year old mixed-breed mare was referred to the equine teaching hospital of the Faculty of Veterinary Medicine in Cluj-Napoca for the evaluation of an extensive, unilateral nasal mass. Upon inspection, a cauliflower-appearing mass was protruding from the right nostril. A decision was made to try to surgically remove the mass using a direct approach through a nasal bone flap. Classic approach through the opening of the nostril was impossible due to the extent of the mass. The surgery was performed with the mare standing under sedation and regional anesthesia. The mass was approximately $30 \mathrm{~cm}$ by $7 \mathrm{~cm}$ and was described histologically as a nasal polyp. The mare made a full recovery, and there was no recurrence in the next three years after surgery.
\end{abstract}

Keywords: equine, surgery; bone flap; nasal polyp.

Received: 31 Dec 2020

Accepted: 16 February 2021

Published: 14 May 2021

DOI:

10.15835/buasvmcn-vm:2020.0049

(c) (1) (3) 2021 Authors. The papers published in this journal are licensed under the Creative Commons Attribution-NonCommercialNoDerivatives 4.0 International License

\section{INTRODUCTION}

The Nasal polyps are not very common in horses, comprising of just about $2,5 \%$ of equine sinonasal disease (Tremaine and Dixon, 2001). However, nasal polyps can develop into extensive masses, obstructing partially or totally air flow thus giving dramatic clinical presentations.

Nasal polyps can be surgically removed with the horse standing, when polyps are inserted more rostrally and are not massive. Several surgical approaches were described: uterine biopsy forceps, urinary catheter snare, bone rongeurs, transendocopic electrocautery. Nasal polyps that are located more caudally can be reached by nasal bone flap or trephination. However, these techniques are usually performed with the horse under general anesthesia. There is another report of a nasal polyp removed in a standing horse, but surgical and anesthesiology approach were different (Schumacher, 2004). In the current case report, the rectangular bone flap generated a good visualization of the mass and enough space for the surgeon to operate, similar to the triangular bone flap technique previously described (Blackford et al 1985). This is important, knowing that profuse hemorrhage can occur during surgery (Nickels, 1993).

The aim of this study was to present a surgical technique used to successfully remove a massive nasal polyp in a standing mare. 


\section{MATERIALS AND METHODS}

A 9 year old mixed-breed mare was referred to the equine teaching hospital of the Faculty of Veterinary Medicine in Cluj-Napoca for the evaluation of an extensive, unilateral nasal mass. The owner reported the mare started having a malodorous nasal discharge several months previously and a significant lack of exercise tolerance. Approximately two months before presentation, a mass was noticeable in the right nostril. The mass grew in size and was protruding about 5 $\mathrm{cm}$ at the time of presentation. No treatment was initiated due to poor prognostic offered by the referring veterinarian.

On admission, a cauliflower appearing mass was protruding from the right nostril (Fig.1 A, B). Airflow was almost completely absent and a malodorous discharge was noticed unilaterally. The mass was mobile and slightly hemorrhagic. No facial deformity was observed and the area was not painful. Endoscopy showed a polypoid multilobulated mass, extended aborally towards the ethmoid and nasopharynx (Fig. 2). The mass was adherent to the nasal septum on a length of $3 \mathrm{~cm}$.

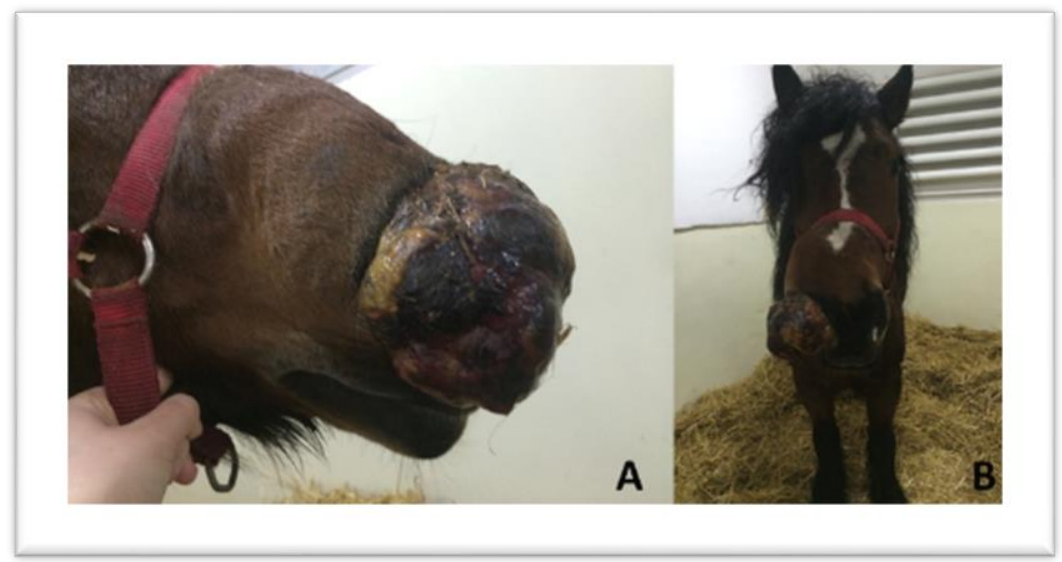

Figure 1. Clinical presentation (A) lateral view, (B) Frontal view

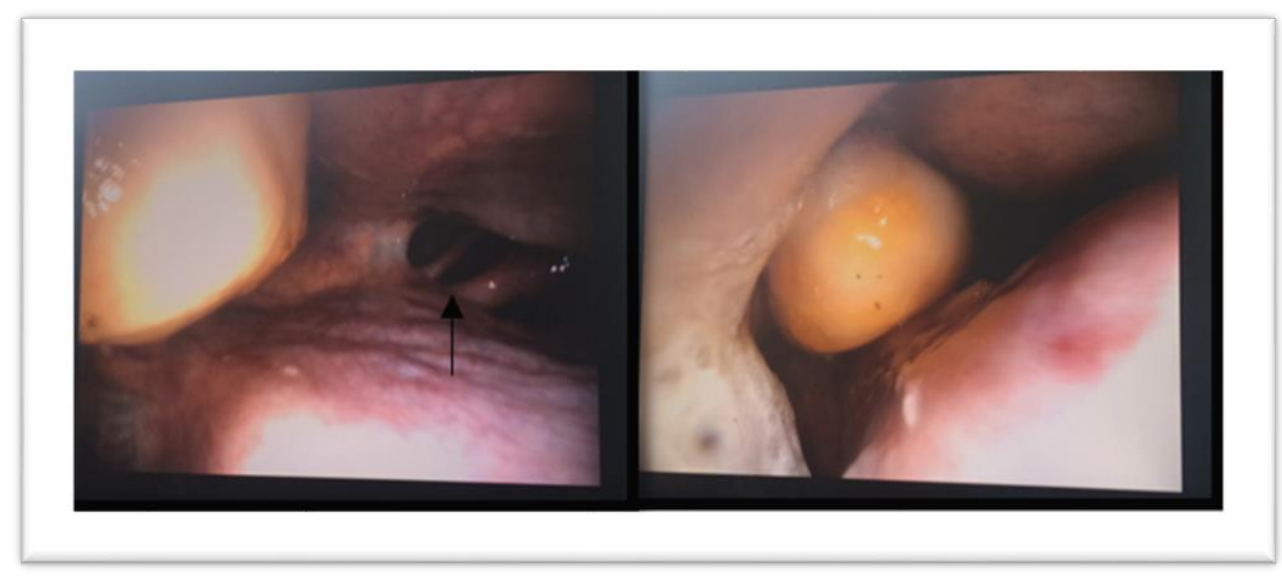

Figure 2. Endoscopic view, right nostril. Black arrow, ethmoid.

\section{Surgery}

The surgery was performed with the mare standing and restrained in stocks. After routine aseptic preparation of the left jugular vein, a $14 \mathrm{G}$ x $133 \mathrm{~mm}$ catheter (High Flow IV Cannula, Fioniavet) was placed. The mare was premedicated with acepromazine maleate $0.025 \mathrm{mg} / \mathrm{kg}$ intravenously (IV) (Trankilrom, Romvac), detomidine $0.01 \mathrm{mg} / \mathrm{kg}$ (Domosedan, Orion Pharma) and butorphanol tartrate $0.02 \mathrm{mg} / \mathrm{kg}$ IV (Butomidor, Richter Pharma). Anesthesia was maintained with a constant rate infusion of detomidine $0,02 \mathrm{mg} / \mathrm{kg}$ IV and butorphanol $0.02 \mathrm{mg} / \mathrm{kg}$ IV diluted in a polyionic isotonic solution (Ringer, B Braun). Regional anesthesia was realized with bupivacaine (Bupivacina 2,5\%, Infomed). The following nerve blocks were performed after aseptic preparation of the area: frontal, infraorbital and maxillary as well as intradermic at the incision site.

A $15 \mathrm{~cm}$ paramedian skin incision was made 5 centimeters above the right nostril (Fig. 3A). Two Gelpi retractors were used for easier access and the periosteum was elevated from the nasal bone. A 5 by $7 \mathrm{~cm}$ incision was made in the nasal bone, using an oscillating bone saw (Fig. 3B). The bone flap was removed together with the nasal mucosa and placed in sterile saline (Fig 3C). The adherent portion of the mass was determined by digital palpation. Two hemostatic forceps were placed at this level, at a $1 \mathrm{~cm}$ distance and the mass was removed by electrocauterization. The bone flap was reattached with the use of surgical wire. Eight holes were bore, one in each corner of the bone flap and one in each corner of the bone 
incision with the use of a $2.4 \mathrm{~mm}$ surgical drill. Periosteum was sutured with a 2-0 Vicryl suture in a simple continuous pattern. Skin was closed with a 2-0 Vicryl suture intradermally (Fig. 4).

Postoperatively the mare was medicated with Flunixin Meglumine $1.1 \mathrm{mg} / \mathrm{kg}$ IV (Niglumine, Dopharma) four days and phenylbutazone $2.2 \mathrm{mg} / \mathrm{kg}$ (Butagran, Dopharma) for the following seven days and a combination of penicillin and streptomycin (Pen Strep, Norbrook) for seven days. Tetanus prophilaxis was made with 6000 units' tetanus toxoid IM (Clostetan, Bioveta).

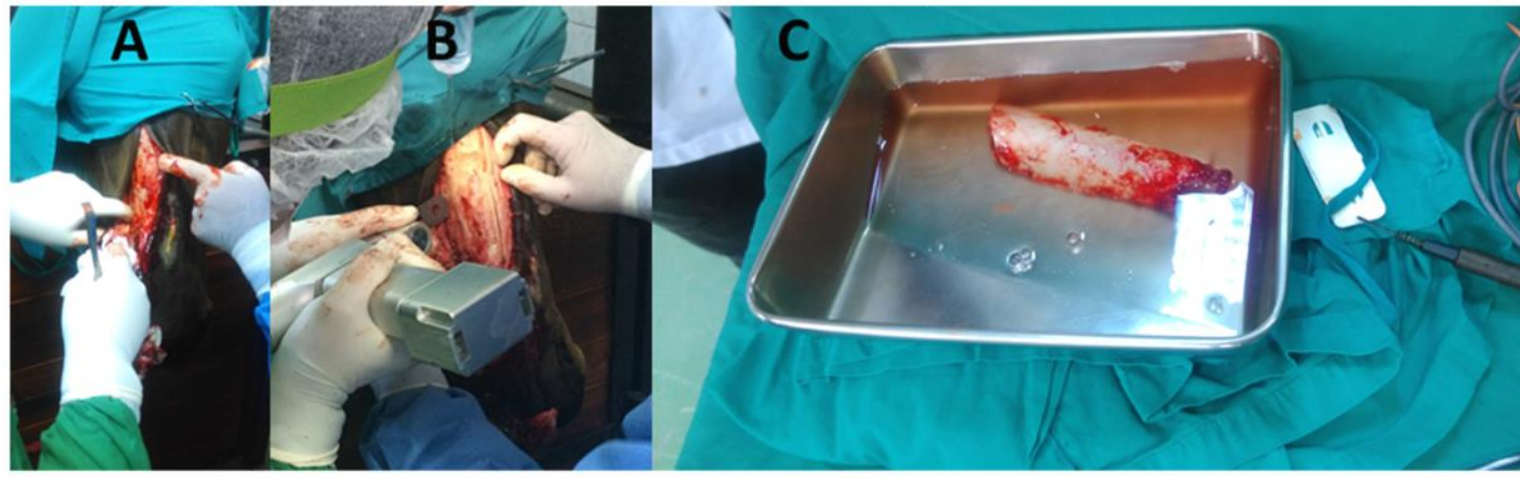

Figure 3. (A) Skin incision; (B) Bone incision; (C) Nasal bone flap

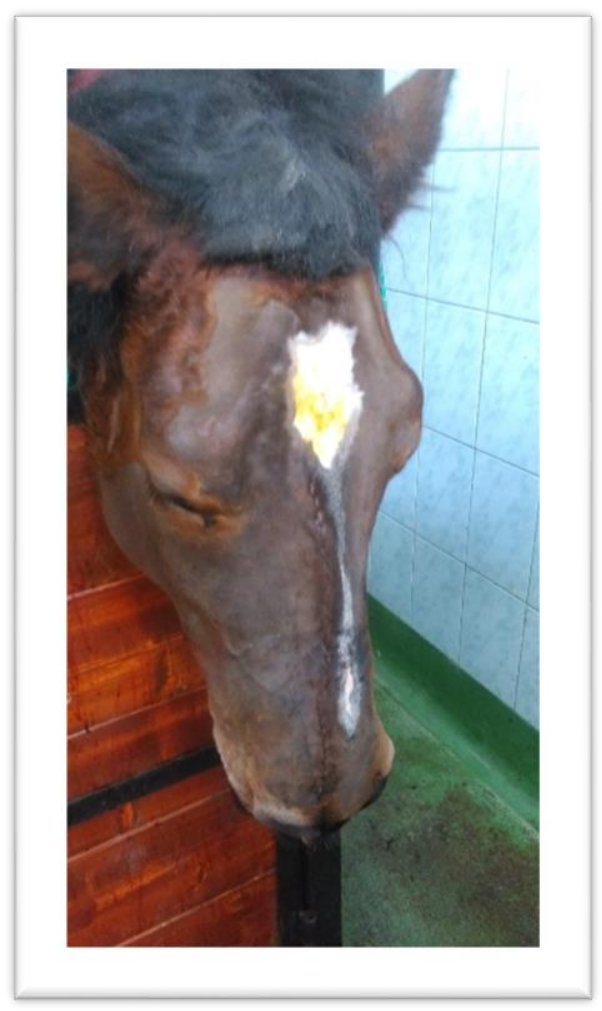

Figure 4. Intradermal skin suture, postoperatively

\section{Tumor characteristics}

Grossly, the polypoid mass was multilobulated, gray-yellow, dense, and showed multifocal areas of necrosis and ulceration associated with hemorrhages (Figure 5). On cross section, abundant white-gray dense fibrous tissue and multiple cystic structures filled with a clear yellow fluid were noticed (Figure 6). The peripheral areas of the polypoid structure were more gelatinous with a myxoid appearance.

Histologically, the exophytic mass contained large amounts of granulation tissue composed of reactive fibroblasts and thick collagen fibers arranged perpendicular to numerous blood capillaries, and was infiltrated with neutrophils, macrophages and lymphocytes (Figure 7). The overlying epithelium was multifocally affected by necrosis and ulceration. 


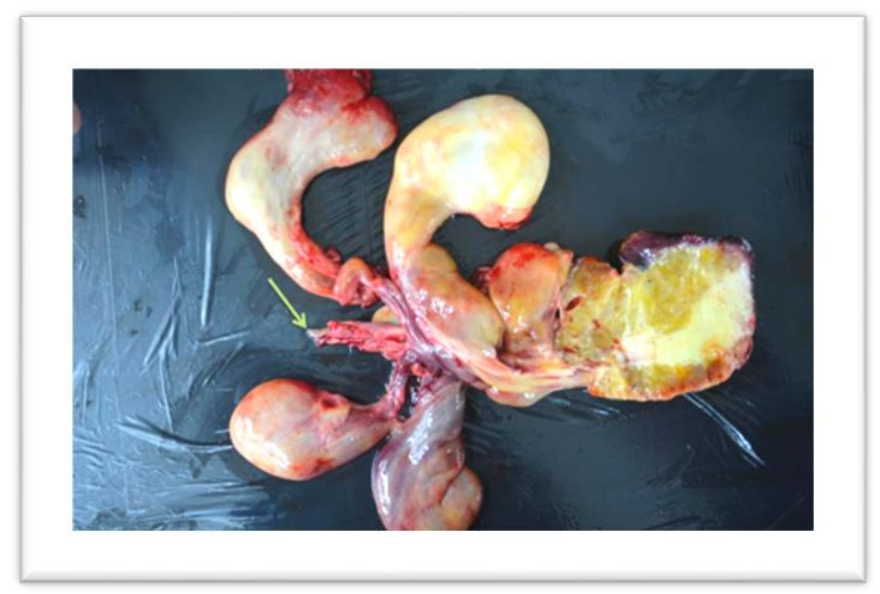

Figure 5. Gross aspect of the mass. Yellow arrow, the part adherent to the nasal septum.

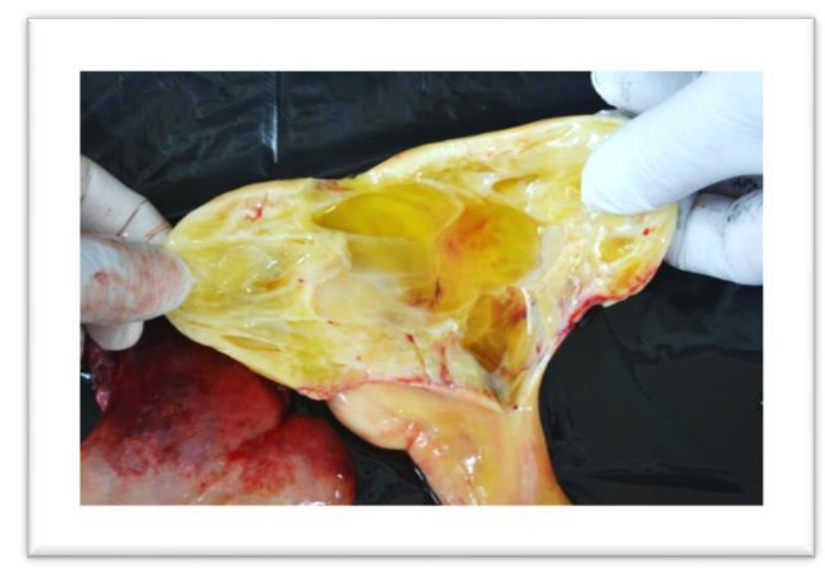

Figure 6. Fluid content of the mass, after incision

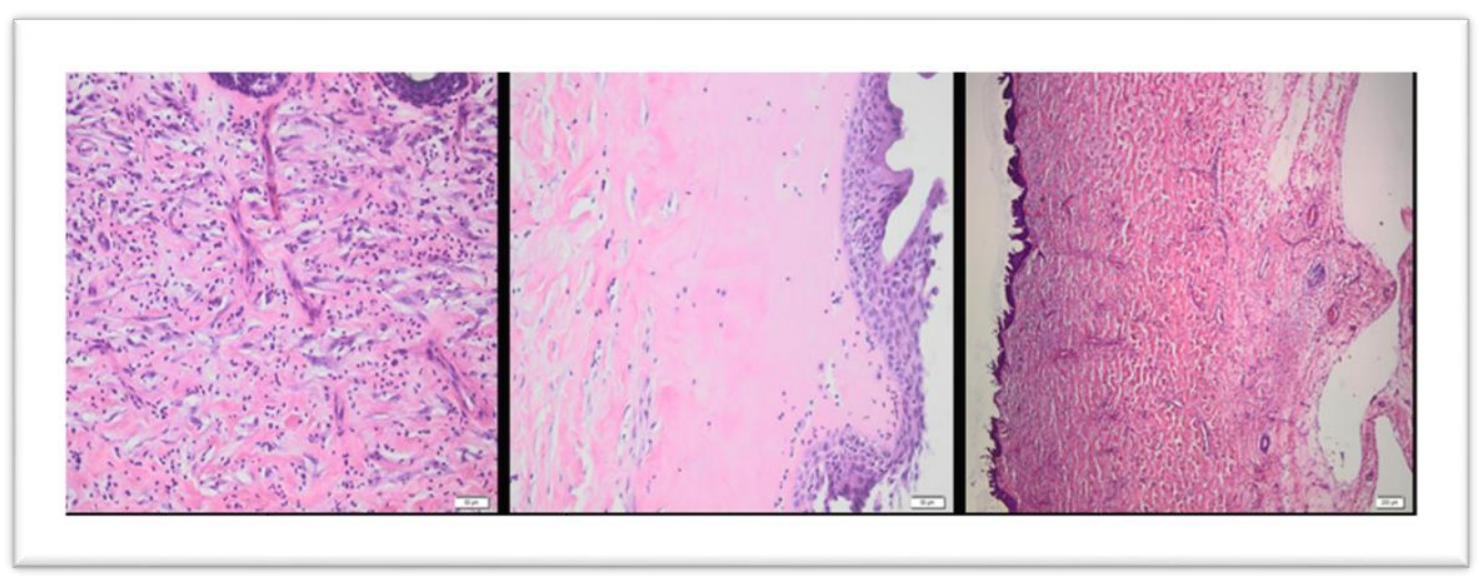

Figure 7. Histological aspects of the mass

\section{CONCLUSIONS}

The mare in this case report had a massive mass in her right nostril that had characteristics both grossly and histologically, consistent with a non haemorrhagic nasal polyp. Nasal polyps are not very common in horses, comprising of just about 2,5 $\%$ of equine sinonasal disease (Tremaine and Dixon, 2001). Nasal polyps are considered to be benign tumors, resulting from an inflammatory overgrowth of a mixomatous network of connective tissue, that includes secretory structures as well (Watt and Beck, 1997). There are two types of nasal polyps, hemorrhagic and non-hemorrhagic (Platt, 1975). In this case, because of the rapid growth and necrotic aspect of the mass, the referring veterinarian suggested euthanasia, and no 
treatment was initiated. Euthanasia is not uncommon in this cases because of clinical presentation that is not improved with antibiotic treatment (Wada et al., 2006).

The mare made a full recovery. A single granuloma was detected 3 months after surgery, at the level of one of the four surgical wires. The granuloma was easily drained and local antiseptic was used for 1 week. At the 3 years follow up, there was no recurrence of the nasal polyp.

Author Contributions: CC and LO performed the surgery, IM designed and supervised the study, MR wrote this case report, CP performed anesthesia, MT interpreted gross and histological aspects.

Funding Source: This research had no funding

Acknowledgments This research did not receive any specific grant from funding agencies in the public, commercial, or not-for-profit sectors.

\section{Conflicts of Interest}

The authors declare that they do not have any conflict of interest

\section{REFERENCES}

1. Blackford JT, D0 Goble, RW Henry, DR Geiser, JP Held. Triangulated Flap Technique for Nasofrontal Surgery Results in Five Horses. Veterinary Surgery. 1985 ; 14, 4, 287-294,

2. Nickels FA. Diseases of the nasal cavity , Vet Clin North Am Equine Pract. 1993; 9 (1): 111-114

3. Platt H. Haemorrhagic nasal polyps of the horse. The Journal of Pathology. 1975: 115(1): 51-55

4. Tremaine WH, Dixon PM. A long-term study of 277 cases of equine sinonasal disease. Part 1: Details of horses, historical, clinical and ancillary diagnostic findings. Equine vet. J. 2001; 33 (3) 274-282

5. Wada E, Akinori S, Masumi S, Takehito M, Kanako S, Kumiko T et al. Horse with Chronic Sinusitis Associated with Empyema and Nasal Polyp, Journal of the Japan Veterinary Medical Association, 2006; 59 (2): 69-72

6. Watt BC, Beck BE. Removal of a nasal polyp in a standing horse. Can Vet J. 1997; 38(2): 108-109 